\title{
On the Process of Accretion in the Formation of the Planets and Comets
}

\author{
J. G. HILLS \\ Department of Astronomy, University of Michigan, Ann Arbor, Michigan 48104
}

Received October 31, 1971; Revised July 3, 1972

The physically reasonable assumption that the seed bodies which initiated the
accretion of the individual asteroids, planets, and comets (subsequently these
objects are collectively called planetoids) formed by stochastic processes requires a
radius distribution function which is unique except for two scaling parameters :
the total number of planetoids and their most probable radius. The former depends
on the ease of formation of the seed bodies while the second is uniquely determined
by the average pre-encounter velocity, $V$, of the accretable material relative to an individual planetoid. This theoretical radius function can be fit to the initial asteroid radius distribution which Anders (1965) derived from the present-day distribution by allowing for fragmentation collisions among the asteroids since their formation. Normalizing the theoretical function to this empirical distribution reveals that there were about $10^{2}$ precollision asteroids and that $V=(2-4) \times 10^{-2}$ $\mathrm{km} / \mathrm{sec}$ which was presumably the turbulent velocity in the Solar Nebula. Knowing $V$ we can determine the scale height of the dust in the Solar Nebula and consequently its space density. The density of accretable material determines the rate of accretion of the planetoids. From this we find, for example, that the Earth formed in about $8 \times 10^{6} \mathrm{yr}$ and it attained a maximum temperature through accretion of about $3 \times 10^{3 \circ} \mathrm{K}$. From the total mass of the terrestrial planets and the theoretical radius function we find that about $2 \times 10^{3}$ planetoids formed in the vicinity of the terrestrial planets. Except for the asteroids the smaller planetoids have since been accreted by the terrestrial planets. About $15 \%$ of the present mass of the terrestrial planets was accumulated by the secondary accretion of these smaller primary planetoids. There are far fewer primary planetoids than craters on the Moon or Mars. The craters were likely produced by the collisional breakup of a few primary planetoids with masses between one-tenth and one lunar mass. This deduction comes from comparing the collision cross sections of the planetoids in this mass range to that of the terrestrial planets. This comparison shows that two to three collisions leading to the breakup of four to six objects likely occurred among these objects before their accretion by the terrestrial planets. The number of these fragments is quite adequate to explain the lunar and Martian craters. Furthermore the mass spectrum of such fragments is a power-law distribution which results in a power-law distribution of erater radii of just the type observed on the Moon and Mars. Applying the same analysis to the planetoids which formed in the vicinity of the giant planets reveals that it is unlikely that any fragmentation collisions took place among them before they were accreted by these planets due to the integrated collision cross section of the giant planets being about three orders of magnitude greater than that of the terrestrial planets. We can thus anticipate a marked scarcity of impact craters on the satellites of these outer planets. This prediction can be tested by future space probes. Our knowledge of the radius function of the comets is consistent with their being primary planetoids. The primary difference between the radius function of the planetoids which formed in the inner part of the solar system and that of the comets results from the fact that the seed bodies which grew into the comets formed far more easily than those which grew into the asteroids and the terrestrial planets. Thus in the outer part of the Solar Nebula the principal 
solid material (water and ammonia snow) accreted into a huge $\left(\sim 10^{12+}\right)$ number of relatively small objects (comets) while in the inner part of the nebula the solid material (hard-to-stick refractory substances) accumulated into only a few $\left(\sim 10^{3}\right)$ large objects (asteroids and terrestrial planets). Uranus and Neptune presumably formed by the secondary accretion of the comets.

\section{INTRODUCTION}

In recent years much effort has been made to understand the formation and structure of the Solar Nebula (e.g. Alfvén, 1954; Hoyle, 1960; Cameron, 1962). In comparison, much less effort and probably less progress has been made to quantitatively understand the physics involved in the growth of the planets and other solar system objects out of the material in the Solar Nebula; although Hoyle (1946), Kuiper (1953), and Hartmann (1968) among others have made notable contributions to aspects of the problem.

It was suggested long ago (ef. Urey, 1952) that the terrestrial planets, asteroids, and the satellites of the giant planets formed by the accretion of grains of refractory materials while Uranus, Neptune, and the comets formed by the accretion of water and ammonia snow. In this paper we will discuss some of the physical aspects of their accretion. A preliminary report on this work has already been given (Hills, 1970). In subsequent discussions we shall collectively refer to all objects formed in the Solar Nebula by the accretion of solid particles as planetoids. Jupiter and Saturn are largely composed of hydrogen and helium (DeMarcus, 1958) which could not have condensed out as solids anywhere in the Solar Nebula; consequently these planets did not form by solid-particle accretion. Their formation is discussed in a companion paper (Hills, 1972).

Appendix A discusses the physical conditions in the Solar Nebula during planet formation. The principal purpose of this discussion is to identify the major types of accretable material in the nebula and their space density as a function of distance from the sun.

The central new idea introduced in this paper is the hypothesis that the seed bodies which initiated the accretion of the individual planetoids formed by stochastic processes. This is the case in most other known processes requiring the formation of seed bodies, and it can reasonably be anticipated to have been true in the Solar Nebula. (A discussion of the formation of the seed bodies is given in Appendix B.) In the remainder of this paper we shall discuss some of the consequences of the hypothesis to check its consistency with available data. We shall also make a special effort to make clear-cut predictions from the hypothesis which can be checked by further experiments and observations.

\section{Radius Function}

The calculation of the radius function of the planetoids requires some knowledge of the rate of formation of the seed bodies that initiated their accretion. If the seed bodies were formed by stochastic processes their rate of formation at any particular point in the nebula remained nearly independent of time as long as the total mass accumulated by the planetoids was much smaller than the amount of unaccreted material.

Under these circumstances the number of planetoids with radii between $R$ and $R+\Delta R$ is directly proportional to the time necessary for the radius of a planetoid lo grow from $R$ to $R+\Delta R$. This requires a radius distribution function of the form

$$
d N(R)=\frac{N_{0}^{\prime} d R}{(d R / d t)}
$$

where the constant of proportionality, $N_{0}{ }^{\prime}$, is the number of seed bodies formed per unit time in the nebula. The rate of growth, $d R / d t$, is determined by the accretion equation (see Hartmann, 1968).

$$
\frac{a d R}{d t}=\alpha \frac{\rho_{a}}{\rho_{p}} \frac{V}{4}\left(1+\frac{8 \pi G R^{2} \rho_{p}}{3 V^{2}}\right) .
$$

Here $\alpha$ is the sticking coefficient, $p_{a}$ is the space density of the accretable material, 
$\rho_{p}$ is the planetoid density, and $V$ is the average pre-encounter velocity of the accreted particles relative to the planetoid. The equation is simplified by introducing a characteristic radius,

$$
R_{c}=\left[3 /\left(8 \pi G \rho_{p}\right)\right]^{1 / 2} V
$$

so that

$$
\frac{d R}{d t}=\alpha \frac{\rho_{a}}{\rho_{p}} \frac{V}{4}\left[1+\left(\frac{R}{R_{c}}\right)^{2}\right]
$$

$R_{c}$ is the radius at which the accretion cross section of a planetoid is twice its geometric cross section.

Making use of the accretion equation, the radius distribution function becomes

$$
d N(R)=\frac{N_{0} d R / R_{c}}{\left[1+\left(R / R_{c}\right)^{2}\right\rceil},
$$

where the new constant of proportionality is

$$
N_{0}=\frac{4 N_{0}^{\prime} R_{c}}{\alpha V} \frac{\rho_{p}}{\rho_{a}} .
$$

Integrating Eq. (5) we find that the number of planetoids with radii equal or less than $R$ is

$$
N(R)=N_{0} \tan ^{-1}\left(R / R_{c}\right) .
$$

As $R \rightarrow \infty$,

$$
N(\infty)=(\pi / 2) N_{0} .
$$

Thus the number of planetoids is formally bound even if the radius of the largest one and the total mass of the system are not. This results from the accretion cross section of the largest object formally growing much faster than its mass which allows it to grow to infinite mass in a finite time if enough material is present. In a real system the number of planetoids is similarly not determined by the total mass of the system but by the ratio of the time necessary for the largest object in the system to acquire most of its mass to the average time between the production of the seed bodies. This is due to the fact that most of the mass in a typical planetoid system is accumulated into the first one or two largest bodies unless the production of the seed bodies has been sufficiently easy that the radius of the largest planetoid is less than $R_{c}$.
In any actual system there is an upper limit, $R_{\max }$, to the radius of the largest planetoid, but if $R_{\max } \gg R_{c}, \quad N(\infty) \sim$ $N\left(R_{\max }\right)$. If we mathematically allow $R \rightarrow \infty$ so that $N(\infty)$ is the total number of planetoids formed, the normalized integrated radius function is

$$
N(\underline{R})=(2 / \pi) N(\infty) \tan ^{-1}\left(R / R_{c}\right)
$$

and in differential form

$$
d N(R)=\left(\frac{2}{\pi}\right) N(\infty) \frac{d R / R_{c}}{\left[1+\left(R / R_{c}\right)^{2}\right]}
$$

We note that $R_{c}$ is the median radius of the planetoids.

We would like to compare this radius function to an empirical one. In most places in the solar system the smaller planetoids have long since been accreted by the planets. The purest population of initial planetoids in the vicinity of the Earth is the asteroids. It is well known that because of their high relative velocities and small masses, collisions among these objects lead to fragmentation rather than coalescence (see Piotrowski, l953). Allowing for this fragmentation Anders (1965) has derived a reconstructed initial radius distribution. Although this only comprises a little more than 100 astcroids and consequently has a large statistical uncertainty as well as the uncertainty introduced by the reconstruction process, Anders finds that when displayed on a log-log plot the distribution can be represented fairly well by a Gaussian with a peak near $R=30 \mathrm{~km}$. This work has basically been substantiated by Hartmann and Hartmann (1968); although Hartmann (1968) notes that a Gaussian distribution underestimates the observed number of more massive asteroids. This suggests that the initial asteroid radius distribution function was broader than a Gaussian, but it still had a distinct bell-like appearance near its peak.

In order to compare the theoretical radius function with the Anders distribution we have to express the former in units of $\ln R$. This yields

$d N(\ln R)=(2 / \pi) N(\infty) \frac{\left(R / R_{c}\right) d\left[\ln \left(R / R_{c}\right)\right]}{\left[1+\left(R / R_{c}\right)^{2}\right]}$ 
This function is plotted in Fig. 1. It is a serpentine curve and looks quasi-Gaussian about the peak at $R=R_{c}$. This fits the reconstructed radius distribution to within the statistical errors if $R_{c} \sim 15 \mathrm{~km}$. This function is noticeably broader than a Gaussian.

For the initial asteroid system the radius of Ceres is $R_{\max }$. We note in passing, that if we normalized the theoretical radius function to the reconstructed asteroid radius distribution, any planetoids with $R>R_{\max }$ predicted by the theoretical relation can only have a mathematical and no physical significance.

With $R_{c}=15 \mathrm{~km}$ and $\rho_{p}=3.6 \mathrm{~g} / \mathrm{cm}^{3}$ for the asteroids we find by Eq. (3) that $V=0.02 \mathrm{~km} / \mathrm{sec}$. The peak of Ander's proposed empirical distribution is $R_{c} \sim 30$ $\mathrm{km}$ (Anders, 1965) with an error of about $50 \%$. For $R_{c}=30 \mathrm{~km}, V=0.04 \mathrm{~km} / \mathrm{sec}$. We take $V-(2-4) \cdot 10^{-2} \mathrm{~km} / \mathrm{sec}$ as the likely range of $V$. This $V$ was presumably due to large-scale turbulent motion in the Solar Nebula.

\section{Total Number of Planetoids}

Assuming that $V$ was constant throughout the Solar Nebula allows the calculation of the total number, $N(\infty)$, of planetoids formed in the principal zones of the Solar Nebula (see Appendix A for a discussion of these). Whether or not $V$ was indeed constant throughout the nebula is best decided on the basis of further experiments, such as from comparing the radius distribution functions of the Trojan asteroids and the comets to the theoretical radius distribution function. The total mass of a system of planetoids in which the largest body has a mass $M_{\max }$, is found by integrating Eq. (10). This gives

$$
\begin{gathered}
M_{\text {total }}=\int_{0}^{R_{\max }}\left(\frac{2}{\pi}\right) N(\propto)\left[\left(\frac{4}{3}\right) \pi R^{3} \rho_{p}\right] \\
\times \frac{d R / R_{c}}{\left[1+\left(R / R_{c}\right)^{2}\right.}
\end{gathered}
$$

On completing the integration and rearranging terms we find

$$
\frac{N(\infty)=}{\left(M_{\max } / M_{c}\right)^{2 / 3}-\ln \left[1+\left(M_{\text {total }} / M_{c}\right)\right.}
$$

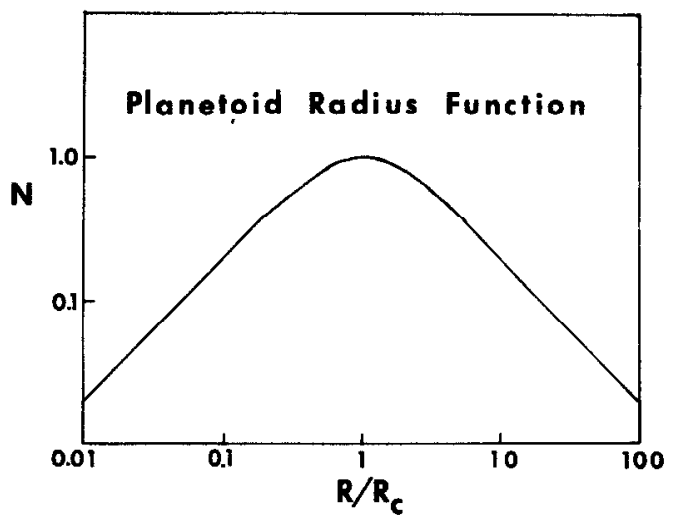

Fig. 1. Theoretical radius function of the planetoids.

where from $\mathrm{Eq} .(3)$

$$
M_{c}=(4 / 3) \pi R_{c}^{3} \rho_{p}=\left[3 /\left(32 \pi G^{3} \rho_{p}\right)\right]^{1 / 2} V^{3} \text {. }
$$

If $M_{\max } \gg M_{c}$

$$
N(\infty) \sim \pi \frac{M_{\text {total }}}{\left[\left(M_{\max }\right)^{2 / 3} M_{c}^{1 / 3}\right]}
$$

In fact typically $M_{\text {total }} \sim 2 M_{\max }$ so

$$
N(\infty) \simeq\left(2^{+2 / 3}\right) \pi\left(M_{\text {total }} / M_{c}\right)^{1 / 3}
$$

For a given $V$ we note that $N(\infty) \alpha \rho_{p}{ }^{1 / 6}$. This weak dependence of $N(\infty)$ on $\rho_{p}$ makes rather immaterial whether we use $\rho_{p}=\mathbf{3 . 6}$ $\mathrm{g} / \mathrm{cm}^{3}$ as for chondrite meteorites or $\rho_{p}=5.5 \mathrm{~g} / \mathrm{cm}^{3}$ as for the Earth or Mercury in calculating $N(\infty)$ for the planetoids which formed in the inner part of the Solar Nebula. In the region of the terrestrial planets (Terrestrial Band) $M_{\text {total }}=2.0 M_{\oplus}$ (total mass of present terrestrial planets and the asteroids) and $M_{\max }=1.0 M_{\oplus}$. In this zone we find from Eq. (13) that if $V=0.02 \mathrm{~km} / \mathrm{sec}, N(\infty)=3508$ for $\rho_{p}=5.5$ $\mathrm{g} / \mathrm{cm}^{3}$ and 3269 for $\rho_{p}-3.6 \mathrm{~g} / \mathrm{cm}^{3}$ while if $V=0.04 \mathrm{~km} / \mathrm{sec}, N(\infty)=1752$ for $\rho_{p}=5.5$ $\mathrm{g} / \mathrm{cm}^{3}$ and 1633 for $\rho_{p}=3.6 \mathrm{~g} / \mathrm{cm}^{3}$. We roughly estimate (see Appendix A) that the planetoids which formed in the vicinity of Jupiter and Saturn (Jupiter-Saturn Band) had $M_{\text {total }} \sim 4 M_{\odot}$ and $M_{\max } \sim 2.5 M_{\oplus}$. For these objects with $\rho_{p}=3.6 \mathrm{~g} / \mathrm{cm}^{3}, N(\infty)=$ 3550 for $V=0.02 \mathrm{~km} / \mathrm{sec}$ and $N(\propto)=1773$ for $V=0.04 \mathrm{~km} / \mathrm{sec}$. We shall discuss the planetoids which formed in the vicinity of Uranus and Neptune later.

Table I tabulates the mass distribution 
TABLE I

Mass Function of the Planetoids (Terrestrial Band) ${ }^{a}$

\begin{tabular}{|c|c|c|c|c|c|c|c|}
\hline \multirow[b]{3}{*}{$\log _{10} \frac{M}{M_{\oplus}}$} & \multirow[b]{3}{*}{$\begin{array}{c}\text { Radius } \\
(\mathbf{k m})\end{array}$} & \multicolumn{6}{|c|}{ Turbulent velocity } \\
\hline & & \multicolumn{3}{|c|}{$V=0.02 \mathrm{~km} / \mathrm{sec}$} & \multicolumn{3}{|c|}{$V=0.04 \mathrm{~km} / \mathrm{sec}$} \\
\hline & & $N_{t}$ & $\sum N_{i}$ & $\frac{\sum \text { Mass }}{M_{\text {total }}}$ & $N_{t}$ & $\sum N_{i}$ & $\frac{\sum \text { Mass }}{M_{\text {total }}}$ \\
\hline-17 & 0.02 & & 2.3 & & & 0.6 & \\
\hline-16 & 0.03 & $\begin{array}{l}2.7 \\
5.8\end{array}$ & 5.0 & & $\begin{array}{l}0.67 \\
1.5\end{array}$ & 1.3 & \\
\hline-15 & 0.07 & 12.5 & 10.9 & & 3.1 & 2.7 & \\
\hline-14 & 0.16 & 27.0 & 23.4 & & 0.7 & 5.8 & \\
\hline-13 & 0.34 & 58.1 & 50.4 & & & 12.6 & \\
\hline-12 & 0.73 & 124 & 108 & $1.6(-11)$ & 31.3 & 27.1 & \\
\hline-11 & 1.58 & 261 & 233 & $2.9(-10)$ & 66.9 & 58.4 & $8.5(-11)$ \\
\hline-10 & 3.41 & 506 & 494 & $6.1(-9)$ & 140 & 125 & I.6(-9) \\
\hline-9 & 7.35 & 756 & 1000 & $1.2(-7)$ & 267 & 265 & $3.2(-8)$ \\
\hline-8 & 15.8 & 699 & 1756 & $1.6(-6)$ & 384 & 533 & $6.1(-7)$ \\
\hline-7 & 34.1 & 422 & 2456 & $1.4(-5)$ & 338 & 916 & $8.3(-6)$ \\
\hline-6 & 73.5 & 210 & 2878 & $8.8(-5)$ & 198 & 1255 & $7.0(-5)$ \\
\hline-5 & 158 & 99.1 & 3088 & $4.5(-4)$ & 97.8 & 1453 & $4.1(-4)$ \\
\hline-4 & 341 & 46.1 & 3187 & $2.1(-3)$ & 46.0 & 1551 & $2.1(-3)$ \\
\hline-3 & 735 & 21.4 & 3233 & $1.0(-2)$ & 21.4 & 1597 & $9.9(-3)$ \\
\hline-2 & 1583 & 9.9 & 3254 & $4.6(-2)$ & 9.9 & 1618 & $4.6(-2)$ \\
\hline-1 & 3410 & 4.6 & 3264 & 0.22 & 4.6 & 1628 & 0.22 \\
\hline 0 & 7346 & & 3269 & 1.00 & & 1633 & 1.00 \\
\hline
\end{tabular}

“ $M_{\max }=1.0 \mathrm{M}_{\bigoplus} ; M_{\text {total }}=2.0 M_{\oplus} ; \rho_{p}=3.6 \mathrm{~g} / \mathrm{cm}^{3}$.

of the planetoids which formed in the Terrestrial Band assuming $\rho_{p}=3.6 \mathrm{~g} / \mathrm{cm}^{3}$ and turbulent velocities of $0.02 \mathrm{~km} / \mathrm{sec}$ and $0.04 \mathrm{~km} / \mathrm{sec}$. Table II shows the corresponding distribution for the planetoids which formed in the Jupiter-Saturn Band. Note that within each of the two zones the mass distribution function is almost independent of $V$ for masses $>10^{-5} \mathrm{M}_{\text {ft }}$. One can show that the number of these massive objects is similarly relatively insensitive to $\rho_{p}$.

\section{ACcreted Planetolds}

Presumably, except for about 100 initial asteroids and their fragments, the smaller planetoids have been accreted by the remaining planets and their larger satellites. From Table I we find that about $15 \%$ of the mass of the original planetoid system 
TABLE II

Mass Function of the Planetoids (Jupiter-Saturn Band) ${ }^{a}$

\begin{tabular}{|c|c|c|c|c|c|c|c|}
\hline \multirow[b]{3}{*}{$\log _{10} \frac{\mathbf{M}}{\mathbf{M}_{\oplus}}$} & \multirow[b]{3}{*}{$\begin{array}{l}\text { Radius } \\
\quad(\mathrm{km})\end{array}$} & \multicolumn{6}{|c|}{ Turbulent velocity } \\
\hline & & \multicolumn{3}{|c|}{$V=0.02 \mathrm{~km} / \mathrm{sec}$} & \multicolumn{3}{|c|}{$V=0.04 \mathrm{~km} / \mathrm{sec}$} \\
\hline & & $N_{i}$ & $\sum N_{i}$ & $\frac{\sum \text { Mass }}{M_{\text {total }}}$ & $N_{i}$ & $\sum N_{i}$ & $\frac{\sum \text { Mass }}{M_{\text {total }}}$ \\
\hline-17 & 0.02 & & 2.5 & & & .6 & \\
\hline-16 & 0.03 & $\begin{array}{l}2.9 \\
6.3\end{array}$ & 5.5 & & $\begin{array}{l}0.7 \\
1.6\end{array}$ & 1.4 & \\
\hline-15 & 0.07 & 13.6 & 11.8 & & 3.4 & 2.9 & \\
\hline-14 & 0.16 & 29.3 & 25.4 & & 7.3 & 6.3 & \\
\hline-13 & 0.34 & 63.0 & 54.7 & & 15.8 & 13.7 & \\
\hline-12 & 0.73 & 135 & 118 & & 34.0 & 29.4 & \\
\hline-11 & 1.58 & 284 & 253 & $1.6 \cdot 10^{-10}$ & 72.7 & 63.4 & $4.6 \cdot 10^{-11}$ \\
\hline-10 & 3.41 & 550 & 537 & $3.3 \cdot 10^{-9}$ & 152 & 136 & $8.5 \cdot 10^{-10}$ \\
\hline-9 & 7.35 & 821 & 1086 & $6.2 \cdot 10^{-8}$ & 290 & 288 & $1.8 \cdot 10^{-8}$ \\
\hline-8 & 15.8 & 760 & 1907 & $8.9 \cdot 10^{-7}$ & 417 & 578 & $3.3 \cdot 10^{-7}$ \\
\hline-7 & 34.1 & 458 & 2666 & $7.8 \cdot 10^{-6}$ & 367 & 995 & $4.4 \cdot 10^{-6}$ \\
\hline-6 & 73.5 & 228 & 3124 & $4.8 \cdot 10^{-5}$ & 215 & 1362 & $3.8 \cdot 10^{-5}$ \\
\hline-5 & 158 & 108 & 3352 & $2.5 \cdot 10^{-4}$ & 106 & 1577 & $2.2 \cdot 10^{-4}$ \\
\hline-4 & 341 & 50.1 & 3460 & $1.2 \cdot 10^{-3}$ & 50.0 & 1683 & $1.1 \cdot 10^{-3}$ \\
\hline-3 & 735 & 23.3 & 3510 & $5.4 \cdot 10^{-3}$ & 23.3 & 1733 & $5.4 \cdot 10^{-3}$ \\
\hline-2 & 1583 & 10.8 & 3533 & $2.4 \cdot 10^{-2}$ & 10.8 & 1757 & $2.5 \cdot 10^{-2}$ \\
\hline-1 & 3410 & 5.0 & 3544 & 0.12 & 5.0 & 1767 & 0.12 \\
\hline 0 & 7346 & & 3549 & 0.54 & & 1772 & 0.54 \\
\hline
\end{tabular}

a $M_{\max }=2.5 M_{\oplus} ; M_{\text {total }}=4.0 M_{\odot} ; \rho_{p}=3.6 \mathrm{~g} / \mathrm{cm}^{3}$.

in the 'Ierrestrial Band was in objects less massive than Mercury which suggests that about $15 \%$ of the mass of the Earth and the other terrestrial planets was accreted as small primary planetoids while the remaining $85 \%$ was accreted as subplanctoid bodies, primarily clumps of dust (see Appendix B). About one-third the mass of these accreted planetoids was in objects having sublunar masses and two-thirds was in objects having masses between that of the Moon and Mercury.

We may be concerned that a collision between a large planetoid in the latter group and a terrestrial planet could cause thoin mutual destruction. Since for such large objects, the gravitational self-binding energy is much greater than the binding energy due to body forces, a breakup is expected if the pre-encounter total kinetic 


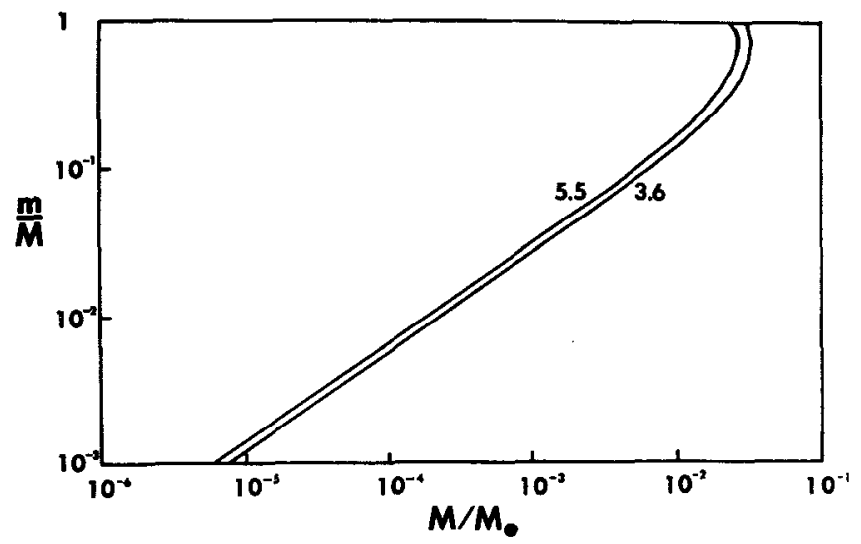

FIG. 2. In a collision between two planetoids having a preencounter relative velocity of $5 \mathrm{~km} / \mathrm{sec}$, $m$ is the minimum mass of the collision partner required to cause the breakup of a planetoid of mass $M$. We note that near the limit of $M$ beyond which a planetoid is stable against breakup irrespective of $m, m$ is double-valued which indicates that there is an upper as well as a lower limit on the $m$ required for a breakup. This results from the quadratic dependence of the gravitational potential energy on $m$ and $M$.

energy, $T$, of the two objects relative to their center of mass is greater than their combined gravitational binding energies, $W$; i.e., for planetoids of uniform density breakup requires that

$$
\frac{1}{2} \mu V^{2}>\frac{3}{5} G\left[\left(M_{1}^{2} / R_{1}\right)+\left(M_{2}^{2} / R_{2}\right)\right],
$$

where $\mu=M_{1} M_{2} /\left(M_{1}+M_{2}\right)$, the reduced mass, and $V$ is the relative velocity of the two objects prior to the encounter. An upper limit on $V$ is probably its present value for interasteroidal collisions, $V=5$ $\mathrm{km} / \mathrm{sec}$ (Piotrowski, 1953). After the terrestrial planets formed, their long-range gravitational perturbations eventually increased $V$ well above the $0.04 \mathrm{~km} / \mathrm{sec}$ maximum turbulent velocity in the Solar Nebula, but these forces were not likely to have had sufficient time before the accretion of most of the small planetoids by the terrestrial planets to raise $V$ much above its present interasteroidal value. Figure 2 shows the mass, $m$, of the smallest body required to cause the collisional breakup of a planetoid of mass $M$ if $V=5 \mathrm{~km} / \mathrm{sec}$ and $\rho_{p}=5.5 \mathrm{~g} / \mathrm{cm}^{3}$ or $3.6 \mathrm{~g} / \mathrm{cm}^{3}$. We note that planetoids more massive than 0.026

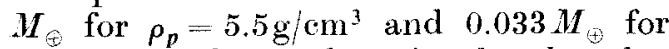
$\rho_{p}=3.6 \mathrm{~g} / \mathrm{cm}^{3}$ are safe against breakup due collisions with all objects equal or less massive than themselves. As the critical mass is less than half that of Mercury (for $\rho_{p}=5.5$ ) it seems likely that all terrestrial planets were safe against collisional breakup. We note that in binding together very small objects, the body forces become more important than gravity and the criteria for breakup are somewhat altered. A discussion of breakup conditions for these objects has been given by Piotrowski (1953) in connection with collisions among asteroids.

Although a collision with a large fellow planetoid would not destroy a terrestrial planet, it would produce a drastic alteration in the direction of its rotational axis if the orbit of the planetoid did not lie in the equatorial plane of the planet. A simple calculation shows that even single collisions with planetoids of lunar mass would easily account for the magnitude of the deviation of the equators of the terrestrial planets from the planes of their orbits. From the theoretical radius distribution function in the Terrestrial Band (Table I) we see that the Earth is likely to have accreted several such bodies.

\section{Collisional Fragmentation of the Plaanetoids}

Their small number $\left[N(\infty) \sim(2-4) \times 10^{3}\right]$ indicates that the accretion of primary planetoids in the Terrestrial Band only 
produced a very small fraction of the observed lunar and Martian craters. As we shall see, likely agents for the production of most craters are collision fragments of a few primary planetoids of approximately lunar mass. We note from Fig. 2 that a lunar-sized object can be broken apart by colliding with a body an order of magnitude less massive than itself while an object the size of Ceres can be broken up by colliding with a body less than two orders of magnitude less massive than itself. This extreme fragility of the asteroids suggests an explanation for their failure to coalesce into one body. If an object of $0.03 M_{\oplus}$ or greater had formed in the asteroid belt, we would likely see only one object today.

The average number of fragmentation collisions that occur among a group of $m$ planetoids before they are accreted by the planets is

where

$$
N=K / \sigma_{p}
$$

$$
K=\sum_{j=1}^{m-1}\left(\sum_{i=j+1}^{m} \sigma_{i j}\right)=\frac{1}{2} m(m-1)\left\langle\sigma_{i j}\right\rangle .
$$

Here $\sigma_{i j}$ is the collision cross section for encounters between planetoids $i$ and $j$, and $\sigma_{p}$ is the total accretion cross section of the terrestrial planets for the average planetoid. With $V=5 \mathrm{~km} / \mathrm{sec}$ the collision cross sections of sublunar planetoids are very nearly their physical cross sections. Thus

$$
\sigma_{i j} \simeq \pi\left(R_{i}+R_{j}\right)^{2} .
$$

Assuming that the radius of the average sublunar planetoid is small compared to the radius, $S_{i}$, of a terrestrial planet, by conservation of energy and momentum we find

$$
\sigma_{p} \simeq \pi \sum_{i=1}^{4} S_{i}^{2}\left[\left(\frac{W_{i}}{\bar{V}}\right)^{2}+1\right],
$$

where $W_{i}$ is the escape velocity from the ith planet. For $V=5 \mathrm{~km} / \mathrm{sec} \sigma_{p}=1.5 \cdot 10^{9}$ $\mathrm{km}^{2}$.

Table I indicates that there were $m=21$ original planetoids in the mass range $0.001-0.01 M_{\theta}$. These have radii between 735 and $1580 \mathrm{~km}$ for $\rho_{p}=3.6 \mathrm{~g} / \mathrm{cm}^{3}$. For these objects $\left\langle\alpha_{i j}\right\rangle \simeq \pi(735+1580)^{2}=$ $1.7 \cdot 10^{7} \mathrm{~km}^{2}$. From Eqs. (18)-(21) we find that $N=2.4$. Thus it is highly likely that two to three fragmentation collisions took place among the objects in this mass range with each collision causing the breakup of two objects.

The observed fragments produced by asteroidal collisions have an integrated radius function of approximately the form (see Hartmann and Hartmann, 1968)

$$
N(R)=\left(R_{\max } / R\right)^{2},
$$

when $N(R)$ is the number of fragments with radii larger than $R$. The largest fragment, of radius $R_{\text {max }}$, has usually about one-half the initial mass of the fragmented planetoid. For the objects we have considered $R_{\max }$ is typically $1000 \mathrm{~km}$ which implies the production of about $10^{6}$ fragments with radii greater than $1 \mathrm{~km}$. This is three orders of magnitude larger than the number of primary planetoids, and it is quite adequate to account for the number of large lunar craters. Because $N(R)$ is very sensitive to $R_{\max }$, we can expect that only the first one or two largest fragmented planetoids produced a majority of all the fragments. This result suggests that while most of the integrated mass of the planetoids and their fragments accreted by a terrestrial planet or the Moon was in the form of a handful of very large unfragmented primary objects, the vast majority of crater-forming bodies were fragments of a few primary planetoids with initial masses on the order of that of the Moon. If meteorites are surviving fragments of planetoids that formed in the vicinity of the Earth rather than fragments which have diffused in from the asteroid belt, we can expect most of them to be from a few primary objects with masses similar to that of the Moon.

Empirical support for this thcorctical picture is provided by studies of the size distribution of lunar and Martian craters (see Hartmann, 1966). These indicate that the bodies which produced the craters had a power-law mass distribution indicative of fragmented objects.

The integrated accretion cross section, $\sigma_{p}$, of Jupiter and Saturn is about three orders of magnitude greater than that of the terrestrial planets. Consequently, Eq. (18) shows that no fragmentation collisions likely occurred among the planetoids that 
formed in the vicinity of the giant planets before these planetoids were accreted by by the planets. Due to the lack of collision fragments the surfaces of the satellites of the giant planets should not be scarred by the large number of impact craters that dominate the face of the Moon and Mars; although, there may be some contamination in the case of the satellites of Jupiter due to the diffusion of fragments from the asteroid belt. Hopefully, this anticipated scarcity of craters can be tested by future space probes.

\section{ThMperature of Accreition}

It is desirable to know whether the temperature which a given planetoid attained during course of its accretion was sufficient to melt it and thereby allow the differentiation of a dense core. The minimum temperature maintained by a planetoid in the act of accretion is one which allows the energy inflow due to accretion to be just balanced by the radiation loss or

$$
\begin{aligned}
\frac{d E}{d t} & =4 \pi R^{2} \sigma\left(T^{4}-T_{0}^{4}\right) \\
& =\frac{G M}{R}\left(4 \pi R^{2} \rho_{p}\right) \frac{d R}{d t} .
\end{aligned}
$$

Using the accretion equation to evaluate $d R / d t$ and by minor algebra we find that

$$
T=\left(\frac{\alpha G \rho_{p} \pi R^{2} V \rho_{a}\left\lceil 1+\left(R / R_{c}\right)^{2}\right\rceil}{3 \sigma}+T_{0}{ }^{4}\right)^{1 / 4}
$$

Here $T_{0}$ is the blackbody temperature of the planetoid in the absence of accretion.

'To evaluate ' $T$ ' for a given planetoid we need to know $\rho_{a}$, the density of accretable material in the Solar Nebula. In Appendix A this has been determined for each of three principal zones in the Solar Nebula. The density is rather sensitive to the turbulent velocity since this determines the scale height of the material perpendicular to the galactic plane. For the Terrestrial Band lying from 0.3 to $2 \mathrm{AU}$ from the Sun we find, for example, that $\left\langle\rho_{a}\right\rangle=1.6 \times$ $10^{-10} \mathrm{~g} / \mathrm{cm}^{3}$ for $V=0.02 \mathrm{~km} / \mathrm{sec}$ and $\left\langle\rho_{a}\right\rangle=$ $0.8 \times 10^{-10} \mathrm{~g} / \mathrm{cm}^{3}$ for $V=0.04 \mathrm{~km} / \mathrm{sec}$. For illustration, in Fig. 3 we have plotted the dependence of $T$ upon $R$ for the planetoids in the Terrestrial Band for a case in which $T_{0}=300^{\circ} \mathrm{K}$ and the planetoid densities, $\rho_{p}$, are $3.6 \mathrm{~g} / \mathrm{cm}^{3}$ and $5.5 \mathrm{~g} / \mathrm{cm}^{3}$. We see that the maximum temperature attained by a planetoid is far more sensitive to $\left\langle\rho_{a}\right\rangle$ than $\rho_{p}$. Eventually we may hope to turn the problem around and form a geological study of each planet, determine the accretion temperature, and consecuently the rate of accretion.

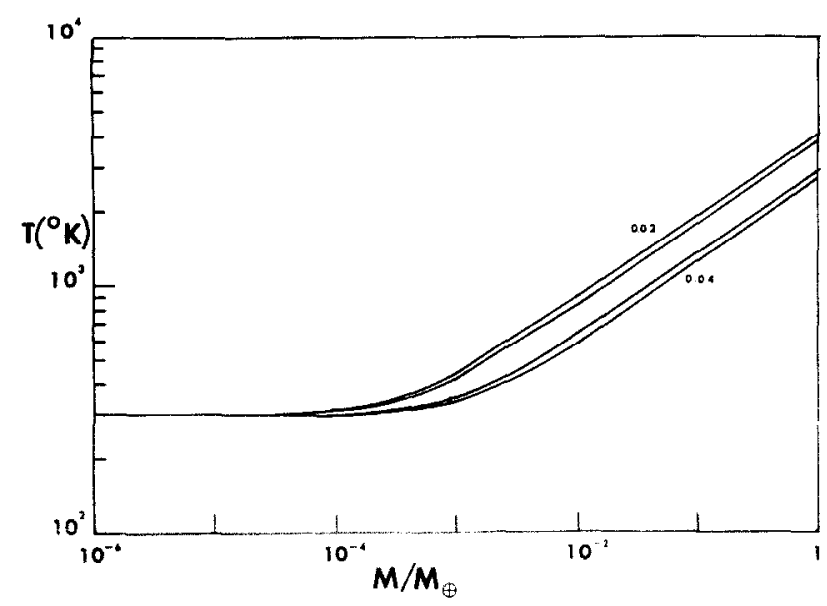

Fra. 3. Maximum temperatures attained by planetoids as the result of accretion. Curves are dramn for turbulent relocities of 0.02 and $0.04 \mathrm{~km} / \mathrm{sec}$. The upper curve in each pair is for $\rho_{p}=5.5 \mathrm{~g} / \mathrm{cm}^{3}$ and the lower is for $\rho_{p}=3.6 \mathrm{~g} / \mathrm{cm}^{3}$. 
We note that if $T \geqslant 2 T_{0}, T$ is practically independent of the particular $T_{0}$ chosen. We further note from the figure that Venus and Earth with $T \sim 3 \cdot 10^{3 \circ} \mathrm{K}$ were possibly the only terrestrial planets which thoroughly melted. Mercury and Mars with $T \sim 1100-1800^{\circ} \mathrm{K}$ may have partially melted. The Moon with $T \sim 600-1000^{\circ} \mathrm{K}$ probably did not melt from accretion, and Ceres with $T \sim 303-320^{\circ} \mathrm{K}$ was essentially accreted cold. Thus an Earth-type core is expected on Venus but probably not on any of the other terrestrial planets. Because of their low accretion temperatures, the asteroids can be expected to have preserved the chemical integrity of the material which they accreted. Thus future on-site inspections of asteroid fragments may yield valuable insight into the chemical and physical properties of the preplanetoid material and consequently insight into the chemical and thermal properties of the Solar Nebula during the time of planetoid formation. Just this type of empirical input is necessary if we are to construct quantitative models of the growth of seed bodies in the Solar Nebula (see Appendix B).

\section{Time of Formation}

If the seed bodies were formed at a uniform rate in time, as has been assumed, in any particular zone of the Solar Nebula the average number of planetoids with radii in some range $R_{1}$ to $R_{2}$ remained constant even in the presence of further accretion as long as the radius $R_{\max }$ of the most massive planetoid in the system was greater than or equal to $R_{2}$. Thus at any given time during the accretion of the planetoids their radius distribution function was the same as given in Tables $I$ and II up to radius $R_{\max }$.

To find the time required for the radius of the largest planetoid in the system to grow to some $R_{\max }$, we integrate Eq. (4). This gives

$$
t=\left[\frac{\left(6 \rho_{p} / \pi G\right)^{1 / 2}}{\rho_{a} \alpha}\right] \tan ^{-1}\left(R_{\max } / R_{c}\right)
$$

We note that, unlike the radius distribution function, this depends on the sticking coefficient $\alpha$, and the space density, $\rho_{a}$, of accretable material. Setting $R_{\max }=\infty$ we see that formally a planet grows to infinite mass in a finite time,

$$
t_{\infty}=\left[\frac{\left(6 \rho_{n} / \pi G\right)^{1 / 2}}{\rho_{a} \alpha}\right] \frac{\pi}{2} .
$$

This is the characteristic time for forming a planetary system. We further note that a planetoid takes only twice as long to grow to $R-\infty$ as to grow to $R-R_{c}$.

Table III tabulates $t$ for planetoids in the Terrestrial Band as a function of $M_{\max }$ for $\alpha=1$ and the previously calculated $\rho_{a}$ 's. The table shows that if $V=0.02$ $\mathrm{km} / \mathrm{sec}$ a planetoid only required about $8 \cdot 10^{4} \mathrm{yr}$ to increase its mass from that of Ceres to that of the Earth. This suggests that if a stable seed body had formed about $8 \cdot 10^{4} \mathrm{yr}$ earlier in the asteroid belt there might be a terrestrial planet there today. This small difference is less than $3 \%$ of the time required for a planetoid mass to grow to $1 M_{\oplus}$.

Table IV shows the time of formation in the Jupiter-Saturn Band. Note that

TABLE III

Time of Growth in the Solar Nebula

\begin{tabular}{|c|c|c|c|c|}
\hline \multirow{3}{*}{$\log _{10} \frac{M_{\mathrm{mux}}}{M_{(\mathrm{B}}}$} & \multicolumn{4}{|c|}{ Time (years) } \\
\hline & \multicolumn{2}{|c|}{$\rho_{p}=3.6 \mathrm{~g} / \mathrm{cm}^{3}$} & \multicolumn{2}{|c|}{$\rho_{p}=5.5 \mathrm{~g} / \mathrm{cm}^{3}$} \\
\hline & $V=0.02$ & $Y=0.04$ & $V=0.02$ & $V=0.04$ \\
\hline-17 & $2.3 \cdot 10^{3}$ & $2.3 \cdot 10^{3}$ & $3.0 \cdot 10^{3}$ & $3.0 \cdot 10^{3}$ \\
\hline-16 & 4.9 & 4.9 & 6.4 & 6.4 \\
\hline-15 & $1.0 \cdot 10^{4}$ & $1.0 \cdot 10^{4}$ & $1.4 \cdot 10^{4}$ & $1.4 \cdot 10^{4}$ \\
\hline-14 & 2.3 & 2.3 & 3.0 & 2.0 \\
\hline--13 & 4.9 & 4.9 & 6.4 & 6.4 \\
\hline-12 & $1.0 \cdot 10^{5}$ & $1.0 \cdot 10^{5}$ & $1.4 \cdot 10^{5}$ & $1.4 \cdot 10^{5}$ \\
\hline-11 & 2.2 & 2.3 & 3.0 & 3.0 \\
\hline--10 & 4.8 & 4.8 & 6.3 & 6.4 \\
\hline-9 & 9.7 & $1.0 \cdot 10^{6}$ & $1.3 \cdot 10^{6}$ & $1.4 \cdot 10^{6}$ \\
\hline-8 & $1.7 \cdot 10^{6}$ & 2.1 & 2.2 & 2.7 \\
\hline-7 & 2.4 & 3.5 & 3.0 & 4.5 \\
\hline 6 & 2.78 & 4.8 & 3.5 & 6.1 \\
\hline-5 & 2.98 & 5.6 & 3.70 & 7.0 \\
\hline$-f$ & 3.08 & 6.0 & 3.81 & 7.4 \\
\hline-3 & 3.12 & 6.16 & 3.86 & 7.63 \\
\hline--2 & 3.14 & 6.25 & 2.88 & 7.73 \\
\hline-1 & 3.151 & 6.28 & 3.895 & 7.77 \\
\hline 0 & 3.155 & 6.30 & 3.900 & 7.79 \\
\hline
\end{tabular}
(Terrestrial Band) 
TABLE IV

Time of Growth in the Solar Nebula (JuPiter-Saturn Band)

Time (years)

\begin{tabular}{|c|c|c|c|c|}
\hline \multirow{2}{*}{$\log _{10} \frac{M_{\max }}{M_{\odot}}$} & \multicolumn{2}{|c|}{$\rho_{p}=3.6 \mathrm{~g} / \mathrm{cm}^{3}$} & \multicolumn{2}{|c|}{$\rho_{p}=5.5 \mathrm{~g} / \mathrm{cm}^{3}$} \\
\hline & $V=0.02$ & $V=$ & $V=0.02$ & $V=0.04$ \\
\hline-17 & $4.4 \cdot 10^{5}$ & $4.4 \cdot 10^{5}$ & $5.8 \cdot 10^{5}$ & $5.8 \cdot 10^{5}$ \\
\hline-16 & 9.5 & 9.5 & $1.3 \cdot 10^{6}$ & $1.3 \cdot 10^{6}$ \\
\hline-15 & $2.0 \cdot 10^{6}$ & $2.0 \cdot 10^{6}$ & 2.7 & 2.7 \\
\hline-14 & 4.4 & 4.4 & 5.8 & 5.8 \\
\hline-13 & 9.5 & 9.5 & $1.3 \cdot 10^{7}$ & $1.3 \cdot 10^{7}$ \\
\hline-12 & $2.0 \cdot 10^{7}$ & $2.0 \cdot 10^{7}$ & 2.7 & 2.7 \\
\hline-11 & 4.4 & 4.4 & 5.8 & 5.8 \\
\hline-10 & 9.3 & 9.4 & $1.2 \cdot 10^{8}$ & $1.3 \cdot 10^{8}$ \\
\hline-9 & $1.9 \cdot 10^{8}$ & $2.0 \cdot 10^{8}$ & 2.5 & 2.6 \\
\hline-8 & 3.3 & 4.0 & 4.3 & 5.3 \\
\hline-7 & 4.6 & 6.9 & 5.8 & 8.9 \\
\hline-6 & 5.4 & 9.5 & 6.8 & $1.19 \cdot 10^{9}$ \\
\hline-5 & 5.8 & $1.09 \cdot 10^{9}$ & 7.2 & 1.36 \\
\hline-4 & 6.00 & 1.17 & 7.43 & 1.45 \\
\hline-3 & 6.09 & 1.20 & 7.53 & 1.49 \\
\hline-2 & 6.13 & 1.22 & 7.58 & $1.5 \mathrm{I}$ \\
\hline-1 & 6.15 & 1.23 & 7.60 & 1.52 \\
\hline 0 & 6.16 & 1.23 & 7.61 & 1.52 \\
\hline
\end{tabular}

because of the lower $\rho_{p}$ (see Appendix A) the characteristic time necessary to form a planetary system in this zone is about two orders of magnitude longer than that in the Terrestrial Band. If the hydrogen and helium in Jupiter and Saturn accumulated on previously accreted planetoid cores as seems to be required (Hills, 1972), it is likely that the Earth and the other inner terrestrial planets formed about $10^{9} \mathrm{yr}$ earlier than Jupiter and Saturn. Thus very substantial amounts of hydrogen and helium gas are likely to have remained in the Solar Nebula until well after the formation of the inner terrestrial planets.

\section{Urants, Neptune, and the Comets}

As we show in Appendix A the planetoids which formed in the region of the Solar Nebula beyond the orbit of Saturn are likely to be composed of a mixture which by mass is about $90 \%$ water and ammonia snow and $10 \%$ refractory material. The two planets in this zone, Uranus and Neptune, are known from their mass-radius relation to be largely composed of $C$, N, and $O$ (Ramsey, 1967) as this would require. Comets apparently are also of this composition (Whipple, 1950); the comets presumably formed in this zone, and subsequently by planetary perturbations, some were ejected into the Oort comet cloud (Oort, 1950).

In our theory comets could either be small primary planetoids or fragments of larger planetoids. We can empirically distinguish between these two possibilities from even our very limited knowledge of the comet radius distribution function. If they are fragments, such as the smaller asteroids, their radius distribution function would be given by Eq. (22). In this case the number of observable comets would increase rapidly with our ability to detect fainter (i.e., smaller) comets. This is the situation for the fainter asteroids, but it is not the case for the comets. Despite the fact that photographs taken with a large telescope can detect objects up to $10^{7}$ times fainter than those observable with the naked eye, the number of new longperiod comets discoverable per year has scarcely increased by even one order of magnitude over that detectable with the naked eye. Thus the comet radius function is clearly not due to fragmentation.

Our knowledge of the plausible comet radius function $i s$ consistent with comets being primary planetoids with $R_{\max }<R_{c}$. Under these circumstances we see from Eq. (5) that the radius distribution function is approximately independent of $R$ which results in the mean $R$ being $\sim 0.5 R_{\max }$ while the average cometary mass $\sim 10^{-1}$ $M_{\max } \cdot M_{\max } \sim 10^{18} \mathrm{~g}$ (Whipple, 1963) which indicates that $R_{\max } \sim 6 \mathrm{~km}$ if $\rho_{p}=1 \mathrm{~g} / \mathrm{cm}^{3}$. The mean comet mass and radius are thus about $10^{17} \mathrm{~g}$ and $3 \mathrm{~km}$, respectively. For $V=0.04 \mathrm{~km} / \mathrm{sec}$ and $\rho_{p}=1 \mathrm{~g} / \mathrm{cm}^{3}, R_{c}=57$ $\mathrm{km}$. So the assumed criterion $R_{\max }<R_{c}$ holds.

The main difference between the radius runction of the comets and that of the planetoids of refractory composition which formed closer to the Sun is that the comet seed bodies formed far more easily than the seed bodies of refractory composition. This 
is hardly surprising; it should have been much easier to bind together snow than rocks! The consequence of this difference is that the water-ammonia snow accreted into a huge number of small planetoids while the refractory material accreted into a few large objects. Most comets were presumably swept up to form Uranus and Neptune while some were tossed out into the Oort comet cloud. The combined mass of Uranus and Neptune is $32 M_{\oplus}$ which indicates they were constructed from about $2 \times 10^{12}$ comets.

Table $V$ shows the rate of growth of planetoids in the Uranus-Neptune Band based on the values of $\rho_{a}$ calculated in Appendix A and a sticking coefficient $\alpha=1$. From this we can see the comets with $R_{\max }=6 \mathrm{~km}$ formed in about $2 \times 10^{8} \mathrm{yr}$ if $V$ was either 0.02 or $0.04 \mathrm{~km} / \mathrm{sec}$. To a close approximation the time required to form objects with $R \ll R_{c}$ is independent of $V$. This results from the fact that $\rho_{a}$ is nearly inversely proportional to $V$ due

\section{TABLE V}

Time of Growth in the Solar Nebula (Uranus-Neptune Band) $\rho_{p}=1 \mathrm{~g} / \mathrm{cm}^{3}$

\begin{tabular}{ccll}
\hline & & \multicolumn{2}{c}{ Time (years) } \\
\cline { 3 - 4 } $\log _{10} \frac{M_{\text {max }}}{M_{\oplus}}$ & $\begin{array}{c}\text { Radius } \\
(\mathrm{km})\end{array}$ & $\begin{array}{l}V=0.02 \\
\mathrm{~km} / \mathrm{sec}\end{array}$ & $\begin{array}{l}V=0.04 \\
\mathrm{~km} / \mathrm{sec}\end{array}$ \\
\hline-17 & 0.02 & $7.6 \cdot 10^{5}$ & $7.6 \cdot 10^{5}$ \\
-16 & 0.05 & $1.7 \cdot 10^{6}$ & $1.7 \cdot 10^{6}$ \\
-15 & 0.11 & 3.6 & 3.6 \\
-14 & 0.24 & 7.7 & 7.7 \\
-13 & 0.52 & $1.7 \cdot 10^{7}$ & $1.7 \cdot 10^{7}$ \\
-12 & 1.13 & 3.6 & 3.6 \\
-11 & 2.43 & 7.7 & 7.7 \\
-10 & 5.23 & $1.6 \cdot 10^{8}$ & $1.7 \cdot 10^{8}$ \\
-9 & 11.3 & 3.4 & 3.5 \\
-8 & 24.2 & 6.2 & 7.2 \\
-7 & 52.3 & 9.3 & $1.3 \cdot 10^{9}$ \\
-6 & 112 & $1.1 \cdot 10^{9}$ & 1.9 \\
-5 & 243 & 1.24 & 2.3 \\
-4 & 523 & 1.29 & 1.5 \\
-3 & 1126 & 1.31 & 2.58 \\
$\cdots 2$ & 2425 & 1.32 & 2.63 \\
-1 & 5226 & 1.33 & 2.65 \\
0 & 11258 & 1.33 & 2.66 \\
& & &
\end{tabular}

to the effect of $V$ on the scale height in the nebula. Under these circumstances we see from Eq. (4) that the rate of growth of planetoids with $R \ll R_{c}$ is independent of $V$. The time required to form the comets was determined primarily by the total mass of accretable material in the UranusNeptune Band. However, the time required to form Uranus and Neptune was dependent on $V$ since for these objects $R \gg R_{c}$. As an example they would have formed in $1.3 \times 10^{9} \mathrm{yr}$ if $V=0.02 \mathrm{~km} / \mathrm{sec}$ or in $2.7 \times$ $10^{9} \mathrm{yr}$ if $V=0.04 \mathrm{~km} / \mathrm{sec}$. It is not unlikely that Uranus and Neptune may only be half as old as the Earth. This suggests the intriguing possibility that there may be planets still in the process of forming by the accretion of comets in the zone beyond the Uranus-Neptune Band since $\rho_{a}$ there would be even lower than in the UranusNeptune Band.

\section{Appendix A : The Solar Nebula Composition and Physical State}

The original composition of the Solar Nebula was presumably the same as in the present-day solar photosphere. We can divide this composition into three principal types of planet-building material: the refractory group, the $\mathrm{CNO}$ group, and the $\mathrm{H}_{2} \mathrm{He}$ group. Of the three groups the refractory material which consists of the heavy elements such as $\mathrm{Mg}, \mathrm{Al}, \mathrm{Ca}, \mathrm{Si}, \mathrm{Fe}$, and their various compounds most easily condensed out of the nebula as solids. This material comprised only about $10^{-3}$ of the original mass of the nebula. The CNO group consisting of the elements $\mathrm{C}, \mathrm{N}, \mathrm{O}$, and their compounds such as $\mathrm{H}_{2} \mathrm{O}, \mathrm{CH}_{\text {, }}$, $\mathrm{CO}$, and $\mathrm{CO}_{2}$ comprised about $10^{-2}$ of the original mass. However, these compounds have much higher vapor pressures than those in the refractory group with the consequence that they were only able to condense out as solids in the outer parts of the Solar Nebula. $\mathrm{H}_{2}$ and He comprising the $\mathrm{H}_{2}$ He group remained gaseous throughout the Solar Nebula. These elements initially accounted for about $99 \%$ of the mass of the nebula, but as noted by Hoyle (1960) they were selectively thermally evaporated from the Solar Nebula especi- 
ally at its periphery. Thus we anticipate a deficiency of these elements in the Solar Nebula during planet formation and consequently in the final planets themselves especially those in the outer parts of the planetary system.

Some theoretical models (see Chiu, 1968) indicate that the Sun may have had a luminosity of about $10^{3} \mathrm{~L}_{\circ}$ when it first reached the quasi-hydrostatic equilibrium stage. From this stage it required about $7.5 \times 10^{7} \mathrm{yr}$ to evolve to the main sequence, but it decreased to its present luminosity in $2 \times 10^{6} \mathrm{yr}$. Since the dust in the nebula rendered it optically thick we can roughly estimate the local temperature of the nebula at some distance $R$ from the Sun by conservation of radiative flux assuming blackbody absorption and emission

$$
T=\left(L / 4 \pi \sigma R^{2}\right)^{1 / 4} \text {. }
$$

With $L=10^{3} \mathrm{~L}, T=2200^{\circ} \mathrm{K}$ at $R=1$ AU and $700^{\circ} \mathrm{K}$ at $R=10 \mathrm{AU}$ while with $L=1 \mathrm{~L}_{\circ}, T=390^{\circ} \mathrm{K}$ and $125^{\circ} \mathrm{K}$ at these two distances. If the nebula were optically thin, the local temperatures would be a factor of $\sqrt{ } 2$ cooler than these calculated values; however, there is good evidence (Hoyle, 1960; Hills, 1972) that these temperatures are lower limits due to probable greenhouse heating of the nebula.

In this picture of pre-main sequence solar evolution, the refractory group was the first material to condense out of the Solar Nebula in solid form. Thus the first objects to grow by accretion were of refractory composition. These planetoids formed throughout the nebula and presumably remain as the asteroids, satellites, terrestrial planets, and the inner cores of the Jovian planets. It may seem difficult to understand how the accretion of the planetoids from the refractory elements could have been initiated since these materials, as commonly found on the Earth have hard, dry surfaces which are not conducive to sticking. This difficulty has been noted by Cameron (1962). This surface property of solids on the Farth results from their having solidified from a liquid phase: however the solids in the nebula likely precipitated directly from a vapor phase which would tend to give them filamentary structures which could intervene during collisions to produce a mechanical binding. In analogy to the case for water, these solids more closely resembled snowflakes which are formed from the vapor phase rather than ice which is formed from the liquid phase.

As shown by Urey (1952) the principal CNO compounds most capable of condensing out of the Solar Nebula were $\mathrm{H}_{2} \mathrm{O}$ and hydrated ammonia, $\mathrm{NH}_{4} \mathrm{OH}$, which together constituted a substantial fraction of the total mass of the CNO compounds. These two compounds condensed out of the nebula at points where the temperature fell below about $140^{\circ} \mathrm{K}$. From the temperature distribution in the nebula deduced by Hills (1972) such temperatures would only have occurred beyond the orbit of Saturn and then only after the luminosity of the Sun had fallen to near its present value. This latter constraint may have allowed the refractory compounds to have condensed out of the nebula up to $2 \times 10^{6}$ yr earlier than any of the CNO compounds. The planetoids which formed inside the orbit of Saturn could only be of refractory composition. The planetoids which formed outside the orbit of Saturn would largely be of CNO composition since this material is about an order of magnitude more abundant than the refractory material. These anticipations are in accord with investigations which indicate that Uranus and Neptune (Ramsey, 1967) and the comets (Whipple, 1950) are of CNO composition.

On the basis of the above discussion we can divide the Solar Nebula into three principal zones of planet formation. The innermost zone from $0.3 \mathrm{AU}$ to $2.0 \mathrm{AU}$ from the Sun we designate as the Terrestrial Band, the intermediate zone from $2 \mathrm{AU}$ to $11 \mathrm{AU}$ is called the JupiterSaturn Band while that from $11 \mathrm{AU}$ to $30 \mathrm{AU}$ is the Uranus-Neptune Band. The specific boundaries of these zones are assigned somewhat arbitrarily. In the inner zones the planetoids are of refractory composition while in the outer zone the planetoids are composed by mass of about $90 \%$ CNO material and 10\% refractory material. In the middle zone the larger 
planetoids were able to accumulate the hydrogen and helium gases to form Jupiter and Saturn (see Hills, 1972).

Some recent computer experiments (Larson 1970) indicate that the sun may not have gone through the high luminosity phase found by earlier workers. In this case the history of some solid material in the Solar Nebula may differ somewhat from that outlined above; e.g., any grains of refractory material present in the original prenebula may have been preserved without evaporation during the evolution of the nebula. However, this does not alter the conclusions concerning the three zones of planet formation. These are based on the types of solid material present in the nebula during planet formation which are the same for the two solar models. The time, $2 \times 10^{6}$ years, required for the sun to sink to its present luminosity in the high-luminosity model is much less than that required to form any of the planets. Thus during planet formation the sun is predicted to have been near its present luminosity by either of the two solar models. Thus the types of solid material available in the Solar Nebula for planet formation is predicted to be nearly the same for the two models.

\section{The Mass of the Planet-Building Materials in the Nebula}

We can estimate the masses and space densities of the three basic planet-building materials in each of the three zones of the Solar Nebula from the present-day masses and compositions of the planets in these zones. In the Terrestrial Band the combined mass of the four inner planets which are almost completcly of refractory composition is $2 M_{\oplus}$. On the basis of relative cosmological abundances this zone is likely to have also contained about $20 \mathrm{M}_{\curvearrowright}$ in CNO gas during planet formation. In the Uranus-Neptune Band the combined mass of Uranus and Neptune is $32 M_{\epsilon \rightarrow}$. On the basis of cosmological abundances we expect about $3 M_{\oplus}$ of this to be refractory material and $29 M_{\oplus}$ of CNO material. The masses of the refractory cores of Jupiter and Saturn are indeterminate, but it is unlikely that they are less massive than the refractory
TABLE VI

Available Planet-Building Materials in the Solar Nebula

\begin{tabular}{|c|c|c|c|}
\hline Zone & Constituent & $\begin{array}{c}\text { Physical } \\
\text { state }\end{array}$ & $\frac{\text { Mass }}{M_{\oplus}}$ \\
\hline \multirow[t]{3}{*}{ Terrestrial } & Refractory & Solid & 2 \\
\hline & CNO & Gas & 20 \\
\hline & $\mathrm{H}_{2} \mathrm{He}$ & Gas & 120 \\
\hline \multirow{3}{*}{$\begin{array}{l}\text { Jupiter- } \\
\text { Saturn }\end{array}$} & Refractory & Solid & 4 \\
\hline & $\mathrm{CNO}$ & Gas & 40 \\
\hline & $\mathrm{H}_{2} \mathrm{He}$ & Gas & 240 \\
\hline Uranus- & Refractory & Solid & 3 \\
\hline \multirow{2}{*}{ Neptune } & CNO & Solid & 29 \\
\hline & $\mathrm{H}_{2} \mathrm{He}$ & Gas & $\sim 0$ \\
\hline
\end{tabular}

material content of Uranus and Neptune. We will arbitrarily assume that the amount of refractory material in Jupiter is $2.5 M_{0}$ and in Saturn is $1.5 M_{\oplus}$ for a combined total of $4 M_{\oplus}$ of refractory material in the Jupiter-Saturn Band. The amount of CNO gas in this zone would then be about $40 M_{\oplus}$. The combined mass of Jupiter and Saturn is about $4.1 \times 10^{2} M_{\oplus}$. Allowing for the other constituents, we estimate that about $3.6 \times 10^{2} M_{\oplus}$ of this is $\mathrm{H}_{2}$ and $\mathrm{He}$. This $\mathrm{H}_{2} \mathrm{He}$ gas was presumably distributed in the Terrestrial and Jovian Bands during the formation of the planetoids. Any $\mathrm{H}_{2} \mathrm{He}$ gas in the Uranus-Neptune zone had a high probability of being thermally evaporated from the Solar Nebula (Hoyle, 1960). The assumption that the amount of $\mathrm{H}_{2} \mathrm{He}$ gas in each of the two inner zones was in proportion to the amount of refractory material in the zone requires $120 M_{\odot}$ of $\mathrm{H}_{2} \mathrm{He}$ in the Terrestrial Band and $240 M_{\oplus}$ in the Jupiter-Saturn Band.

In Table VI we summarize the expected masses of the basic planet-building materials in each of the three zones. In order to determine the average space density of these building materials we must determine their scale heights above the plane of the nebula.

\section{Scale Heights in the Solar Nebula}

The average scale height of a particular group of particles in the nebula depends on 
their average velocity. If the velocities are thermal, the run of density $\rho$ with height, $h$, above the plane follows the usual barometric law.

$$
\rho=\rho_{0} \exp \left[-\beta \mu \int_{0}^{h} g d h\right],
$$

where $\mu$ is the molecular weight, $g$ is the component of the local surface gravity perpendicular to the plane of the nebula, $\rho_{0}$ is the density at $h=0$, and

$$
\beta=(k T)^{-1}=\left(1 / 3 \mu V^{2}\right)^{-1} .
$$

Here $T$ is the temperature, $k$ is Boltzmann's constant, and $V^{2}$ is the mean squared velocity of the particles. The mass of the nebula is sufficiently low that $g$ is essentially just the component of the solar force perpendicular to the plane. Thus

$$
\begin{aligned}
& \rho(r, h)= \\
& \quad \rho_{0} \exp \left\{-\beta \mu\left[\frac{G M_{0}}{r_{0}}\left(1-\frac{r_{0}}{\left(r_{0}^{2}+h^{2}\right)^{1 / 2}}\right)\right]\right\},
\end{aligned}
$$

where $r_{0}$ is the distance from the Sun to a point in the plane of the nebula, and $h$ is the height above this point. The height (scale height) at which $\rho=e^{-1} \rho_{0}$ is

$$
h_{0}=r_{0}\left[\left(1-\frac{r_{0}}{\beta \mu G M_{\odot}}\right)^{-2}-1\right]^{1 / 2} \text {. }
$$

Using Eq. (27) to evaluate $T$, we can now determine the scale height as a function of $r_{0}$ in the nebula. This is tabulated in Table VII for $L=1 \mathrm{~L}_{0}, \mu=2$ and $18 \mathrm{AMU}$, the average molecular weights for $\mathrm{H}_{2}$ and $\mathrm{H}_{2} \mathrm{O}$, respectively. The mean molecular weight of $\mathrm{H}_{2} \mathrm{O}$ is typical of the molecules formed from the CNO group of elements.

The thermal velocities of the dust grains are negligible; however we can anticipate some macroscopic nonthermal motion in the gas and consequently in the dust dragged along by it. The velocity of such mass motion is likely to considerably exceed the Brownian velocity of the dust. Such motion could for instance be due to turbulence resulting from differential rotation (von Weiszäcker, 1944) or possibly from convection. The theory of the Solar Nebula structure is too primitive to permit
TABLE VII

Scale Heights in Solar Nebula

(AU)

\begin{tabular}{rcccc}
\hline & & & \multicolumn{2}{c}{ Dust } \\
\cline { 4 - 5 }$R_{0}$ & $\mathrm{H}_{2}$ & $\mathrm{H}_{2} \mathrm{O}$ & $v=0.02$ & $v=0.04$ \\
& & & & \\
\hline 0.3 & 0.013 & 0.0045 & 0.00009 & 0.00018 \\
0.5 & 0.026 & 0.0085 & 0.00019 & 0.00039 \\
0.8 & 0.046 & 0.0153 & 0.00039 & 0.00078 \\
1.0 & 0.061 & 0.0202 & 0.00055 & 0.00110 \\
2.0 & 0.145 & 0.0481 & 0.00155 & 0.00310 \\
5.0 & 0.455 & 0.1512 & 0.00613 & 0.01226 \\
10.0 & 1.084 & 0.3598 & 0.01734 & 0.03469 \\
20.0 & 2.582 & - & 0.04905 & 0.09811 \\
30.0 & 4.292 & - & 0.09012 & 0.18024 \\
& & & & \\
\hline
\end{tabular}

us to calculate $V$, but by comparing the observed and theoretical radius function of the asteroids we found that $V=0.02-0.04$ $\mathrm{km} / \mathrm{sec}$ at least in the vicinity of the asteroids. Knowing $V$ we can by Eq. (29) evaluate the characteristic $\beta$ of the dust. From $\beta$ we can then, by Eq. (31) determine the scale height, $h_{0}$, of the dust. This is tabulated in Table VII for $V=0.02$ and $0.04 \mathrm{~km} / \mathrm{sec}$. This large-scale motion also kept the dust well-mixed so that the accretion by a planetoid did not lead to an appreciable local dectease in the dust density until the planetoid had grown to about terrestrial-planet size.

\section{Density}

The a verage density, $\rho_{a}$, in the plane of the nebula of a particular class of particles is given closely by

$$
\rho_{a}=\frac{m}{V}=m /\left[2\left(2 \pi \int_{R_{1}}^{R_{2}} r h_{0} d r\right)\right],
$$

where $h_{0}$ is the local scale height, $m$ is the total mass of the particles in the annulus having an inner radius $R$, and outer radius $R_{2}$, and $V$ is the characteristic volume of the annular sector. Equation (32) was intecrated numerically to find the densities shown in Table Vill. In these calculations we have used the masses given in Table VI. For the characteristic scale height of the ONO group of elements (where gaseous) 
TABLE VIII

Densities in the Solar Nebula

$\left(10^{-10} \mathrm{~g} / \mathrm{cm}^{3}\right)$

\begin{tabular}{llllllll}
\hline & & CNO & \multicolumn{2}{c}{ Solid } & & \multicolumn{2}{c}{ Total } \\
Region & $\mathrm{H}_{2} \mathrm{He}$ & (Gas) & $v=0.02$ & $v=0.04$ & $v=0.02$ & $v=0.04$ \\
\hline Terrestrial & 0.96 & 0.48 & 1.6 & 0.80 & 3.04 & 2.24 \\
Jupiter-Saturn & 0.0074 & 0.0038 & 0.0082 & 0.0041 & 0.0194 & 0.0153 \\
Uranus-Neptune & - & - & 0.0020 & 0.0010 & 0.0020 & 0.0010 \\
\hline
\end{tabular}

we have used that of $\mathrm{H}_{2} \mathrm{O}$ and for the $\mathrm{H}_{2} \mathrm{He}$ group we have used that of $\mathrm{H}_{2}$.

\section{Appendix B: The Physical Nature of THE SeEd Bodies}

It was proposed as a hypothesis in the main body of this paper that the protoplanetoid seed bodies formed by a stochastic process with the consequence that their rate of formation was time independent. The principal justification for any hypothesis (as opposed to just a deduction from previously proposed theories, hypotheses, or commonly accepted notions) is its ability to correctly tie together bits of empirical data which previously have appeared unrelated and more importantly to make predictions which can bo tosted by further experiments. If these experiments confirm the predictions we can at least use the theory as a good working hypothesis. The hypothesis proposed in this paper does tie together a number of previously unrelated facts, and it does make predictions which can only be tested by more experiments. While its worth must depend primarily on the correctness of its predictions, we shall demonstrate its reasonableness by deduction from generally assumed notions and hypotheses concerning the conditions in the Solar Nebula. In the rest of this section we shall qualitatively discuss the growth of bodies in the Solar Nebula up to the size of seed bodies.

Small grains were dragged along quite effectively by the turbulent cddies in the Solar Nebula. The relative velocities among such bodies in collisions were on the order of the Brownian velocity rather than the turbulent velocity of $20-40 \mathrm{~m} / \mathrm{sec}$ found in the main body of the paper. As a loose agglomerate of grains built up by collisions its inertial mass increased faster than its surface area (and consequently its drag) with the result that it became progressively more uncoupled from the motion by the eddies. We can understand the physics of the situation better by considering a point mass in the plane of the nebula moving in a circular orbit around the sun. From its vantage point the direction of the wind produced by the motion of the eddies changes on a time scale of the order of or less than its orbital period. When the direction of the wind changes the inertia of a grain resists the velocity change. Consequently it feels a sudden burst of wind which decreases in speed as the drag reduces the difference in velocity between the grain and the eddy. The more massive the body the longer it takes to match its velocity to that of the eddy and the longer and stronger the average bursts of wind produced by such velocity differences. Finally, if a body could grow to a radius $R_{2}$, a meter or so in diameter, its motion becomes uncoupled from that of the individual eddies owing to the time required for it to be picked up by an eddy being longer than the time between changes in the velocity of the eddy. The average wind velocity felt by such an object due to the motion of the eddies is just the turbulent velocity of $20-40 \mathrm{~m} / \mathrm{sec}$ found in the main text.

The wind flowing past a large clump of 
accreted grains carries with it grains and smaller grain clumps which impact the body. If the original grain clump or agglomerate is sufficiently sturdy it tends to gain mass by the accretion of these smaller bodies, but the agglomerate is a very loosely packed, fragile structure which may just as easily be sand-blasted apart as built up by the impacting grains. If the agglomerate does increase in mass it needs to be progressively sturdier to survive the greater average impact velocities of the bodies swept into it by the cddics. In this type of physical situation we can expect the loose agglomerates to grow fairly easily and nearly monotonically up to a characteristic radius for which the impact velocities have become sufficiently energetic that a typical agglomerate is just as easily broken down as built up by the impacting bodies. This radius $R_{1}$, depends on the nature and strength of the binding forces among the bodies in the agglomerate.

Growth beyond radius $R_{1}$ tends towards the destruction of the agglomerate due to the progressively more energetic impacts. However, a few agglomerates being luckier and sturdier than their fellows will be able to acquire larger radii. The production of objects with radii much greater than $R_{\text {: }}$ will be a stochastic process with the number of objects falling off very quickly with increasing radius. A very few of these will acuire a radius $R_{2}$, sufficiently large that they become uncoupled from the motions of the eddies. These objects are the seed bodies of the planetoids since once an object has become uncoupled from the eddies a further increase in mass no longer affects the velocity of the bodies impacting it. Thus if an object is sturdy enough to grow up to radius $R_{2}$ it should grow monotonically in size at a rate governed asymptotically by the accretion equation (4) of the main text.

The closer $R_{1}$ is to $R$, the more seed bodies are formed per unit time. Thus based on the empirical evidence one infers that in the Uranus-Neptune Band where numerous comets formed $R_{1} \lesssim R_{2}$ while in the Terrestrial Band where the formation of seed bodies was difficult $R_{1} \ll R_{2}$.
Detailed quantitative calculations of the growth of objects up to the size of seed bodies is beyond the scope of this paper and not a little dangerous due to the lack of a sufficiently detailed empirical handle on conditions in the Solar Nebula. This is especially true of the determination of $R_{1}$. Its value depends on the nature and strength of the binding among bodies within a loosely packed grain clump. These bodies are likely of heterogeneous composition and structure which makes the calculation of the binding strength espccially tricky. It is also possible that the strength of these bonds are timedependent; e.g., bodies initially bound together by mechanical intertwining may eventually become cold welded. Because of the ease of forming objects up to radius $R_{1}$ we can anticipate that most of the mass accreted by a planetoid to be in bodies with radii near $R_{1}$. Thus we predict that most of the mass accreted by a planetoid was in the form of loose grain clumps a few centimeters across.

With $R_{1} \ll R_{2}$ these grain clumps were transported very easily by the eddies, and their density scale height was the same as calculated in Appendix A. However, when an object has grown up to seed body size $R_{2}$, or greater it should remain very nearly in the plane of the Solar Nebula.

To summarize, we see that the proposed hypothesis which states that the formation of the seed bodies was due to a stochastic process rather than an accumulative process with the consequence that their rate of formation was time-dependent, certainly follows by deduction of $R_{1} \ll R_{2}$. Of course, other factors may lead to the same result without our simplified picture of preseed body construction being correct in detail. Our purpose has only been to demonstrate the reasonableness of the hypothesis. Its validity is best assessed by the degree of conformity between its predictions and the results of further experiments.

\section{References}

ALFVEN, H. (1954). "On the Origin of the Solar' System." Oxford University Press, London. 
ANDERs, E. (1965). Fragmentation history of asteroids. Icarus 4, 399 408 .

Cameron, A. G. W. (1962). The formation of the sun and planets. Icarus 1, 13-69.

Chiv, H. (1968). "Stellar Physics," Vol. 1, p. 476. Blaisdell, Waltham, Massachusetts.

De Marcus, W. C. (1958). The constitution of Jupiter and Saturn. Astron. J. 63, 2-28.

HartmanN, W. C. (1966). Martian cratering. Icarus $5,565-576$.

HartmanN, W. K. (1968). Growth of asteroids and planetesimals by accretion. Ap. J. 152. 337-342.

Hartmand, W. K., and Hartmann, A. C. (1968). Asteroid collisions and evolution of asteroidal mass distribution and meteoritic flux. Icartes $8,361 \cdots 381$.

HiLls, J. G. (1970). The formation of the terrestrial planets. Bull. Amer. Astron. Soc. 2, 320.

Hills, J. G. (1972). On the formation of the hydrogen-rich planets. Icarus, submitted.

Hoyle, F. (1946). On the condensation of the planets. Mon. Not. Roy. Astron. Soc. 106. 405-422.

Hoyle, F. (1960). On the origin of the Solar
Nebula. Quart. J, Roy. Astron. Soc. 1, 28-55.

KUTPER, G. (1953). Note on the origin of the asteroids. Proc. Nat. Ac. Sci. 39, 1159-1167.

LaRson, R. B. (1969). Numerical calculations of the dynamics of a collapsing protostar.

OORT, J. H. (1950). The structure of the cloud of comets surrounding the solar system, and a. hypothesis concerning its origin. Bull. Astron. Inst. Neth. 11, 91-110.

Protrowski, S. (1953). The collisions of asteroids. Acta Astron., Ser. A, 5, 115-136.

RAMSEY, W. H. (1967). On the constitution of Uranus and Neptune. Planet. Spac. Sci. 15, 1609-1623.

Urex, H. C. (1952). "The Planets." Yale University Press. New Haven.

VoN Weizsäcker, C. F. (1944). Zeit. Astrophys. $22,319$.

Whipple, F. L. (1950). A comet model I. The acceleration of Comet Encke. Ap.J. 375-394.

Whipple, F. L. (1963). On the structure of the cometary nucleus. In "The Solar System" (B. M. Middlehurst and G. P. Kuiper, eds.), Vol. 4, Chap. 19. Univ of Chicago Press, Chicago. 